\title{
Comprensión Empática y Estilos de Negociación en la relación de pareja Herramientas de mediación
}

\author{
Dra. Maria Isabel Aguilera Varela ${ }^{1}$
}

\begin{abstract}
RESUMEN
En México y en otros lugares del mundo existen una serie de ideas populares, mitos y creencias múltiples, a cerca de cuestiones relativas al amor y a la pareja, expresadas en conceptos como la media naranja, la renuncia propia para poder amar, la complementariedad necesaria en la pareja, para que esta relación se suponga como posible.

Ideas que provocan mucho sufrimiento en las personas que mantienen una relación de pareja y ven como sus expectativas iniciales no se cumplen. Una vez pasado el momento inicial del enamoramiento, descubren que algo ha pasado o con su relación, o con todos aquellos conceptos sobre las relaciones que habían escuchado hasta en canciones, en algún momento.

En la relación de pareja, la manera como se manejan los conflictos, el estilo de vínculo o relación, la aceptación o negación de la alteridad soportable o insoportable del compañero/a, las distorsiones en la comunicación, el estilo de negociación utilizado en la resolución de los conflictos existentes y la visibilidad del otro, son aspectos que hoy se vuelven a revisar, dada la complejidad de la cultura de la cooperación, auto composición o mediación.
\end{abstract}

\section{PALABRAS CLAVES}

Mediación, Negociación en la relación de la pareja, Comprensión Empática, Herramientas de mediación

\begin{abstract}
In Mexico and in other places of the world there exist a series of popular ideas, myths and multiple beliefs, to near questions relative to the love and to the pair expressed in concepts as the orange average, the proper resignation to be able to love, the necessary complementarity in the pair in order that this relation is supposed like possibly.

Ideas that provoke a lot of suffering in the persons who support a relation of and see as your initial expectations are not fulfilled. Once spent the initial moment of the falling in love, they discover that something has happened or with your relation, or with all those concepts on the relations to that they had listened even $\mathrm{n}$ songs, in some moment. In the relation of pair, the way like they handle the conflicts, the style of link or relation, the acceptance or denial of the bearable or unbearable of the companion, the distortions in the communication, the style of negotiation used in the resolution of the existing conflicts and the visibility of other one, are aspects that today they return to check, given the complexity of the culture of the cooperation, autocomposition or mediation.
\end{abstract}

\footnotetext{
${ }^{1}$ Universidad del País Vasco. Correo electrónico: centromandragora@ prodigy.net.mx
} 


\section{KEYWORDS}

Mediation, Negotiation in the relation of the pair, Empathic comprehension, Tools of mediation

\section{INTRODUCCIÓN}

En la convivencia de pareja, la forma de comunicarse, el estilo de negociación utilizado en la resolución de los conflictos existentes y la visibilidad del otro, son aspectos que hoy se vuelven a revisar. Branden (2000) explica como una relación intima en la que nos sentimos plenamente percibidos por otra persona siempre implica en algún momento, la percepción de unas capacidades de las que no éramos conscientes, de una potencialidad latente, de rasgos que nunca ascendieron al nivel de la percepción explícita. La visibilidad implica a menudo, el descubrimiento de uno mismo.

En lo específico a la psicología de la pareja, Carter y Sokol (2000) se han percatado de que para crear y mantener un verdadero vínculo, los dos miembros de la unión deben esforzarse por darse a conocer, cuestión bien diferente a la de compartir rituales y relaciones mediante estereotipos y conductas adquiridas.

Dentro del ámbito de la terapia de pareja es frecuente observar como a los miembros de una relación, se les dificulta incluso un diálogo en que ambos puedan comprender el punto de vista de su interlocutor.

Los distintos autores expertos en negociación y conflicto, suelen proponer una estrategia basada en la consideración de ambas partes, como salida funcional a un conflicto interaccional.

Así, en un afán por contribuir con una revisión teórica a desarrollar alguna ayuda de carácter preventivo para complementar el trabajo terapéutico, se llevó a cabo la siguiente investigación, que tuvo como objetivo determinar en que medida el grado de comprensión empática en el vínculo de pareja puede estar relacionado con la forma de negociar. 


\section{JUSTIFICACIÓN}

Los conceptos de comprensión empática y los distintos estilos de negociación que son de interés para este proyecto de investigación, han sido abordados desde distintos paradigmas o "círculos epistemológicos", en diferentes momentos históricos.

El concepto de empatía ha evolucionado desde la figura sobresaliente C.Rogers (1985), hacia concepciones actuales que resaltan su funcionalidad en el manejo de conflictos desde el punto de vista de la comunicación, de la subjetividad, o de la calidad de la relación desde una perspectiva emocional, intercultural o como parte de la sinergia o inteligencia social. Así mismo, hoy se considera una herramienta en la solución negociada de conflictos.

Recientemente Armenta, (2002) ha descrito como para la teoría centrada en la persona, el recorrido terapéutico significa ir facilitando una reestructuración o reacomodación de las experiencias, de manera que el si mismo pueda expandirse para poder aceptar todas aquellas vivencias que se han rechazado, desplazado o han tenido una simbolización inadecuada.

Por otro lado, Velasco Alva (2004) desde un modelo psicodinámico constructivista, realiza una propuesta intersubjetiva, empleando la empatia y la identificación proyectiva, a partir del eje transferencia y contratrasferencia. Explicando los vínculos humanos mediante ambas, en donde ésta es vista como un proceso identificatorio transitorio que permite comprender los estados emocionales del otro, así como un mecanismo necesario para la recepción del material del paciente.

Desde las herramientas para la mediación, Plutchik en 1992 considera la empatía como un proceso en el que dos o más individuos comparten experiencias emocionalmente significativas. Sin atribuir un rol cognitivo para las emociones, lo que hace que la falta de la misma esté en la base de los conflictos de relaciones personales. (Citado en Redorta, 2004)

De acuerdo con Rogers, el concepto de empatía forma parte de lo que éste autor consideró como fuente de conocimiento, en el que intervienen tanto un marco de referencia interno, la empatía en sí misma y un marco de referencia externo. Desde su punto de vista, es importante el papel del aprendizaje significativo o vivencial, en el cual intervienen tanto los aspectos cognitivos como los afectivos, siendo este aprendizaje mucho más que una mera acumulación de datos.

El grado de comprensión empática puede ir desde un nivel inicial de empatía que no se corresponde con los sentimientos y experiencia del otro implicando una patente falta de respeto, permitiendo una conversación en términos generales, vagos y anónimos o manteniendo despegado al otro, sin autodescubrimiento de los sentimientos o de la personalidad. Hacia un nivel más profundo en donde el grado de empatía exige mucha atención y respeto profundo, ayudando a expresar matices y multitud de sentimientos o experiencias personales de modo específico, concreto, global, con indicios de respuestas auténticas de modo constructivo.

C.Rogers, comenzó hablando de la labor del terapeuta como un reflejar empático, para acabar concibiéndolo como un encuentro interpersonal, de la mano de la congruencia. 
Más adelante, sobre las base de las condiciones terapéuticas descritas por Rogers, G.T. Barret-Lennard (1962) desarrolló un inventario de la relación que evaluase el grado en que dichos elementos se hayan presentes, incluyendo distintos elementos de la calidad de la entrevista y la relación de ayuda, entre otros el nivel de aprecio.

Para este autor, es valioso evaluar la percepción en la relación, del grado en que dichos elementos se hayan presentes como un intento de profundizar en el proceso terapéutico.

Por su parte, los modelos cognitivos o motivacionales, se centran en la motivación altruista o egoísta para la conducta de ayuda. La mayoría de las veces separan el componente afectivo y cognitivo en el concepto, incluyendo la simpatía como parte de los componentes de la empatía.

Más recientemente, el interés en la solución de conflictos desde una perspectiva intercultural, se refiere a la empatía como una competencia alocéntrica que condiciona particularmente la capacidad de comunicar. Lo que incluye la necesidad de considerar aptitudes como la suspensión del yo, la consideración hacia el otro y la disposición para entrar en contacto, la flexibilidad y tolerancia psicológica y la comprensión del otro en sus necesidades, sentimientos y opiniones. Marandón, (2003).

Las teorías sociales del desarrollo moral abordan el concepto de empatía, en las figuras de Kolhberg (1975) y Piaget (1962), como la habilidad de ver las cosas desde la perspectiva del otro, proponiendo un enfoque constructivista y socio-cognitivo en el cual el desarrollo de la identidad está determinado por el desarrollo moral.

Por su parte, hoy en día, dada la complejidad de la cultura de la cooperación o autocomposición se habla del modelo de Harvard como aquella negociación colaborativa asistida por un tercero, dirigida a la satisfacción de intereses o necesidades que son incompatibles. A modo de conflicto en donde se conducirá a un camino de transacción, basado en el acuerdo entre las partes.

El modelo transformativo, se basa en un enfoque terapéutico propuesto para potenciar el cambio en las personas, a partir de las relaciones humanas desde sus propias habilidades. Caminando hacia el crecimiento moral, la revalorización y el reconocimiento de cada persona. No busca tanto el acuerdo. Se basa en la comunicación y las relaciones interpersonales entre las partes.

El modelo narrativo impulsado por Sara Cob, parte de las aportaciones de M. White proponiendo un análisis de la historia alternativa, teniendo en cuenta las relaciones, el contexto cultural, los mitos, los valores, la incidencia en la identidad y en el self. Pone el acento en la comunicación y en la interacción entre las partes. Redorta (2004), Muñoz Hernán (2003), Díez Tapia (2006).

El concepto de comprensión empática y los distintos estilos de negociación, constituyen una posibilidad de describir la calidad de una relación intersubjetiva y por otro lado valorar los costes y beneficios de una negociación que implica un pronóstico a corto y largo plazo en el mantenimiento de una relación de pareja. 


\section{METODOLOGÍA}

Muestra. Participaron 50 sujetos concertados de manera individual con el único criterio de que en el momento de aplicar el inventario mantuvieran una relación de pareja, encontrándose en distintas etapas de la relación. El 54\% eran mujeres y el $46 \%$ hombres con una edad que fluctuó entre los 17 y 52 años, y el tiempo de relación en pareja iba de 1 año hasta 35 años.

\section{Instrumentos}

1. Se partió del Inventario de la relación de ayuda de G.T Barret-Lennard, en su versión OS-M_64, adaptado al español por A.Celis (1998), con marco teórico en C. Rogers. A partir de las preguntas del inventario correspondientes al grado de comprensión empática, se elaboró un cuestionario tipo likert, escogiendo los 16 ítems relacionados con la misma, añadiendo otros 16, invirtiendo la dirección de la pregunta. Los primeros referidos al grado en que en la relación de pareja se percibe empatia y los siguientes al grado en que se manifiesta empatia.

2. Se tomó el Inventario de estilos de negociación elaborada por Reyes (2002) que consiste en un cuestionario tipo likert con 48 reactivos que evalúa los tres estilos básicos de negociación: distributivo, posicional (duro y suave) e integrativo. El cual se había sometido a una validación por jueces con estudiantes de maestría de la materia "Negociaciones Internacionales" del ITAM.

3. Se añadió una ficha de identificación con las variables de clasificación sexo, edad, escolaridad, ocupación y tiempo de relación en pareja.

\section{Procedimiento}

Se requería contactar con personas con características particulares y se procedió a solicitar el acceso a una escuela de educación especial nivel de primaria, y a una empresa privada donde era factible contactar a personas que en el momento de la aplicación tuvieran pareja.

Antes de iniciar se preguntó a las personas si deseaban participar explicándoles que era un inventario sobre pareja y que la información seria manejada de manera confidencial. La aplicación se realizó de manera individual y en promedio los participantes tardaron un promedio de 30 minutos en contestar.

\section{RESULTADOS}

Los resultados de la investigación fueron analizados aplicando una t de Student y el estudio Anova para conocer la existencia de diferencias significativas entre grupos. También se realizó un análisis de correlación de Pearson, para responder a la cuestión de si existe una relación entre el grado de comprensión empática en la relación de pareja y el uso de diferentes estilos de negociación, dependiendo de su sexo, escolaridad, ocupación y tiempo de relación.

La comprensión empática se define como el grado en el cual una persona es consciente de lo que en este momento le ocurre a otra persona internamente. Como un intento activo por conocer enteramente lo que vivencia, un esfuerzo por captar su comunicación y el significado de ésta y por traducir sus palabras y señales en significados experienciales que coincidan el menos con aquellos aspectos que le son 
más importantes en este momento. Un percibir lo que vivencia el otro "detrás" de lo que comunica explícitamente, pero teniendo presente que este vivenciar se origina y procede del otro. Celis (1999).

El estilo de negociación Distributivo, Coercitivo o de suma cero es aquel proceso donde lo que un negociador gana, significa la perdida del otro. Se obtiene mediante estrategias de dominar al oponente a través de tácticas aceptadas socialmente y otras no aceptadas o "sucias" para lograr su propósito.

El estilo de negociación por posiciones o posicional se define como aquel proceso en donde los negociadores toman posturas de duro o suave para lograr su propósito en el acuerdo. Donde a veces lo más importante es la postura del negociador más que los intereses que se están acordando, motivando que lo que se gane es defender la propia postura personal en lugar de los puntos o intereses en disputa en la negociación con independencia de perjudicar a la otra parte. O no llegando a nada en el proceso. Se denomina también transacción o regateo.

El estilo de negociación integrativo, denominado colaborativo, con base en los méritos o basado en intereses es aquel proceso donde las personas son resolutotas de problemas a través de conocer los intereses y características de la otra parte y proponer alternativas que satisfagan esos intereses de acuerdo a la perspectiva de cada negociador. Reyes (2002).

Se establecieron los factores de la investigación, a partir de los cuales se realizó un diseño no experimental correlacional, lo que se muestra en la figura 1.

\section{Figura 1. Diseño de la investigación}

A continuación se presentarán en primer lugar los resultados de la investigación respecto a la existencia de diferencias significativas, y en segundo, el análisis del estudio de correlación entre las variables.

Como se puede observar en la gráfica 2, no se encontraron diferencias significativas en función del sexo, ni en la manera de percibir y manifestar empatia, como en el uso de los distintos estilos de negociación., utilizando todos ellos de manera similar.

\section{Gráfica 2. Distribución por sexo}

La mayoría de estudios confirman una diferencia en relación al género en la empatia a favor de las mujeres. El modelo de aflicción de Hoffman, reporta estudios en donde hombres y mujeres difieren menos cuando se contempla la empatía como un constructo congnitivo, como una toma de perspectiva.

Es posible que el nivel de comunicación de los participantes sea el mismo. La comunicación puede tener lugar en cuatro niveles diferentes del menor al más productivo. Diferenciando entre diatriba, discusión, debate, o un diálogo que consiste en hablar uno con otro siendo mucho más satisfactorio. Cuando el diálogo progresa, ambos interlocutores están conectados tanto emocionalmente como mentalmente y el resentimiento y la frustración dejan paso a la esperanza y 
afabilidad. Ambos miembros de la pareja sienten cual es la postura del otro y se interesan por lo que experimenta. Houston y Goldberg (2002).

Marándon (2003) desde el punto de vista intercultural, Redorta (2004), y Moore (1995) describen como la confianza se vincula a la tipologia del conflicto y como es necesario cultivar inicialmente un clima de confianza, para poder empatizar.

Respecto al grado de empatia que se percibe y manifiesta en la relación de pareja y el empleo de los distintos estilos de negociación distributivo, posicional o integrativo en función de la escolaridad o dependiendo de la ocupación, los resultados obtenidos son similares. Lo que sugiere la idea de lograr realizar un estudio con diferentes niveles de ocupación y escolaridad debido a que en la presente investigación las personas participantes tenían un nivel análogo siendo la mayoría profesionistas, psicólogos y maestros como se manifiesta en la tabla 3.

\section{Tabla 3. Distribución por ocupación.}

Al respecto Rivera Aragón y Díaz Loving (2002), informan de investigaciones recientes en donde parece darse un cambio en el cual

las mujeres al obtener una escolaridad de licenciatura, aprenden otra manera de afrontar el conflicto, buscando un poder más asimétrico en las relaciones.

Díaz Guerrero (1982, citado en Rivera Aragón y Díaz-Loving), explica como las personas con mayor escolaridad tienden a alejarse de la cultura tradicional que prescribe formas diferentes para hombres y mujeres, cambio que se debe al aumento de la participación femenina en el ámbito laboral, situación que en algunas de sus investigaciones se ha presentado.

Jung (1923) describió un uso constructivo de las diferencias y como se manifiesta el ego a través de los tipos psicológicos, explicando cuatro grados de crecimiento en el hombre y en la mujer. Rogers (1985) asignó un papel relevante al aprendizaje significativo. Así, los sujetos participantes en esta investigación pueden estar ubicados en un proceso de crecimiento psicológico equivalente como afirman Piaget (1962) y Kolhberg (1975) respecto al desarrollo moral.

El tiempo de relación en pareja no causó impacto respecto a las variables estudiadas.

Se observó una ligera tendencia a usar un estilo de negociación posicional en aquellos sujetos que se encuentran en la etapa de diferenciación y realización con un tiempo de convivencia de 8 a 14 años. Esto coincidió con los resultados que encontró Reyes (2002) en donde se encontraban diferencias en el estilo de negociación empleado dependiendo del número de años de vivir juntos. La seguridad en sí mismo en la resolución de conflictos aumenta ligeramente de los cuatro a los siete años de vivir juntos, aunque la tendencia es a ir disminuyendo según aumenta el tiempo de convivencia en pareja.

Podría ocurrir dadas las características de la muestra, que ésta sea la tendencia o que llegue un punto en la relación en donde los individuos comienzan a ceder en etapas posteriores.

Resulta muy interesante el análisis del ciclo básico del conflicto que ha realizado Redorta (2004) considerando que no se trata solo de ver el proceso en el tiempo, sino de entender que un conflicto tiene una dinámica en parte previsible y que 
seguirá un esquema central de tensión, culminación y distensión o en otras palabras escalada, estancamiento y desescalada. Cuando una relación de poder se sostiene muy igualada y la balanza coste/beneficio de mantener el conflicto comienza a cuestionarse porque se ha mantenido en el tiempo o porque consume muchos recursos y energía se llega al estancamiento como punto critico del proceso en donde se puede pasar de actividades competitivas a actividades colaborativas y se reconoce al otro. Redorta (2004).

El grupo que utiliza un estilo de negociación integrativo confiando en la mutualidad de la pareja para resolver conflictos sin ceder, de una manera creativa lo constituyo el conformado por los sujetos que se encontraban en la etapa denominada de reafirmación como pareja y paternidad que transcurre de los cuatro a los siete años de convivencia. En relación a los hijos Winter (1973) y Díaz Loving (2002) afirman que pueden ser una variable importante en las tácticas de poder de una persona así como en la manera de cómo es expresado pudiendo canalizar o dirigir el modelo de poder hacia una forma socialmente responsable. También explican como existen parejas en matrimonios duraderos que utilizan estrategias diferentes que aquellas parejas de corta duración.

Redorta (2004) describe los ejes de la escalada de un conflicto manifestando como al inicio se pueden utilizar tácticas suaves incrementando la coerción hasta llegar a tácticas más duras ampliando los problemas en este proceso y desplazando los problemas hacia las personas surgiendo una motivación individual egoísta y competitiva, acabando finalmente por implicar a terceros.

Ponti (2005) resume los costes y beneficios de una negociación posicional e integradora y su nexo con los resultados a corto y largo plazo en el mantenimiento de las relaciones.

Respecto al análisis de correlación se encontró que cuanto más considera el sujeto que es percibido empáticamente, menos uso hace de una negociación colaborativa. El hecho de que en la relación de pareja se considere que se percibe el punto de vista del sujeto se convierte en un elemento de desconfianza a la hora de resolver los conflictos con mutualidad.

Lo que puede comprenderse ya que el uso de una negociación colaborativa suele generar sospechas. Usualmente suele pensarse que sólo se colabora en los cuentos de hadas, con lo que se percibe la negociación desde una perspectiva competitiva, con desconfianza. Ponti (2005).

Jota (1998) relata que el uso de una negociación posicional suele ser el método más habitual en la resolución de conflictos de pareja. Lo cual no es de buen pronostico y a la mayoría de los acuerdos se debe llegar regateando. En este estilo el negociador desea evitar los conflictos personales con lo que o hace concesiones de inmediato o ve cualquier situación como una disputa de voluntades en donde la parte que toma las posiciones más duras o extremas durante más tiempo, obtiene más.

A medida que la confianza desciende en una relación, las actitudes pasan de ser cooperativas a competitivas y basadas en la desconfianza mutua. Los implicados en 
el proceso tienden a maximizar sus ganancias o minimizar sus pérdidas. Redorta (2004).

La otra cara de la estrategia cooperativa es su vulnerabilidad a la explotación lo que explica la mayor desconfianza en que las soluciones se busquen entre los dos.

En la presente investigación el grado de empatia que percibe el sujeto lo lleva a usar más un estilo de negociación posicional. La empatia se convierte en un elemento de seguridad y confianza personal a la hora de enfrentar los conflictos aunque con el riesgo de generar una estrategia que se convierte en un ganar-perder.

Rivera Aragón y Díaz Loving (2002) se refieren a un estudio de Scanzoni en función del tipo de matrimonio, en donde la negociación de poder entre los esposos puede ser mayor cuando la esposa incrementa sus recursos por medio del empleo siendo más propensa a retos maritales en papeles, reglas y arreglos negociando por un balance igual de poder dentro del hogar.

Es posible que se de una negociación posicional dependiendo de las áreas de conflicto y una negociación colaborativa si se trata de preservar la relación en el tiempo como afirma la propuesta situacional, cuestión que se puede investigar.

Como se aprecia en la figura 3 los sujetos de este estudio cuanto más utilizan un estilo de negociación posicional como elemento de seguridad personal partiendo del conocimiento de la pareja para enfrentar los conflictos, usan más un estilo de negociación colaborativo.

\section{Figura 4. Análisis de correlación}

Esto sugiere la idea expresada por Muñoz Hernán de que existen dos actitudes en la negociación. La actitud posicional dura implica la defensa de la propia postura personal y la tendencia a ganar, deslindándose de los riesgos de explotación que conlleva una actitud más integrativa. Es posible que al aprender a defender su postura personal adquieran una experiencia en el manejo de la negociación, sus beneficios y costes, que los lleve a desarrollar el mayor uso de una negociación colaborativa, lo cual se ha relacionado con la asertividad necesaria en este estilo, en donde se manifiesta mayor confianza en resolver los conflictos entre los dos, aceptando las diferencias.

Es necesario un buen análisis del conflicto implícito en la discrepancia. Aprender a negociar, puede que implique atravesar las distintas caras de la negociación, desarrollando las distintas facetas mas funcionales o disfuncionales. Como indica Sánchez (2003) a veces es necesario recorrer todo el ciclo del conflicto. Identificar los desencadenantes y partir de ciertas hipótesis de trabajo previas, que permitan cuestionar las actitudes, formas de conducta o la relación estructural de los litigantes. Lo cual puede servir de pronóstico en la resolución de conflictos, de costes y beneficios en los resultados y en la calidad de las relaciones de convivencia mantenidas en el tiempo. 


\section{CONCLUSIONES FINALES}

El concepto de comprensión empática es una variable importante en el estudio de la intersubjetividad, de la calidad de una relación y el establecimiento de relaciones más significativas. La investigación es reciente y sugiere tendencias, sin embargo abre nuevos interrogantes.

La investigación presente, con sus límites, confirma la hipótesis de trabajo que planteaba una posible relación entre el grado de comprensión empatica y el uso de diferentes estilos de negociación. Aunque no se pueden extrapolar los resultados y son necesarias muestras más amplias y diversificadas, se observó una correlación entre el grado de compresión empatica y el empleo de estilos de negociación específicos.

Además los individuos pueden salir fortalecidos si son capacitados en ponerse en lugar del otro, establecer un diálogo y aprender a relacionarse de una forma significativa y negociar a favor de la relación. Por lo que se realiza una propuesta de nuevas líneas de investigación e incide en la necesidad de realizar un trabajo terapéutico con las parejas que incluya los elementos socioafectivos de reconstrucción en la mediación de conflictos comunitarios y la negociación más profunda cuando un conflicto está polarizado y afecta a los miembros de una relación de pareja.

\section{BIBLIOGRAFÍA}

Armenta, J. (2001). Empatía y Psicoterapia: Las vicisitudes del acompañamiento centrado en la persona. Revista de Psicología Humanista y Desarrollo Humano, Prometeo, 28, 60-63.

Branden, N (2000) La psicología del amor romántico. Paidós. México.

Carter, S, Sokol, J. (2000). Del Amor al Compromiso. Para alcanzar una relación de pareja estable. Barcelona. Ediciones Urano.

Celis, H. (1999). Proceso de Adaptación del Inventario de la Relación de Ayuda de G.T. Barrett-Lennard. Revista de Psicología de la Universidad de Chile, Vol VIII, $N^{\circ} 1$, Santiago de Chile.

Diez, F, Tapia, G. (2006) Herramientas para trabajar en mediación. Paidós Mediación. Buenos Aires. Cuarta reimpresión.

Marandón, G. (2001). Más allá de la empatía hay que cultivar la confianza: claves para el encuentro intercultural. Revista Cidob D'affers Internacionals, 61-62. MayoJunio.

Redorta (2004). Como analizar los conflictos. La tipologia de los conflictos como herramienta de mediación. Barcelona Paidós Mediación.

Reyes, T (2002) Relación entre estilos de negociación en la pareja y la satisfacción de necesidades afectivas. Tesis de Maestría. COPHAC. México. 
Rivera, A.S y Díaz-Loving, R (2002). La cultura del poder en la pareja. Facultad de Psicología. UNAM. México.

Rogers, C. (1985) Terapia, Personalidad y Relaciones Interpersonales. Buenos Aires. Nueva Visión.

Moore, C. (1995). El proceso de mediación. Métodos prácticos para la resolución de conflictos. Barcelona. Granica.

Muñoz- Hernán, Y. (2003). Buscando respuestas. ¿Qué es la mediación y la negociación? ¿Por qué esta de moda? ¿Cómo se hace? Disponible en: www.gernikagogoratuz.org

Velasco, Alva, F. (2004). Parejas en Conflicto, Conflictos de Pareja: Manual Psicodinámico Constructivista para su tratamiento. ETM. México. 
Apéndice

\section{Figura 1. Diseño de la Investigación}

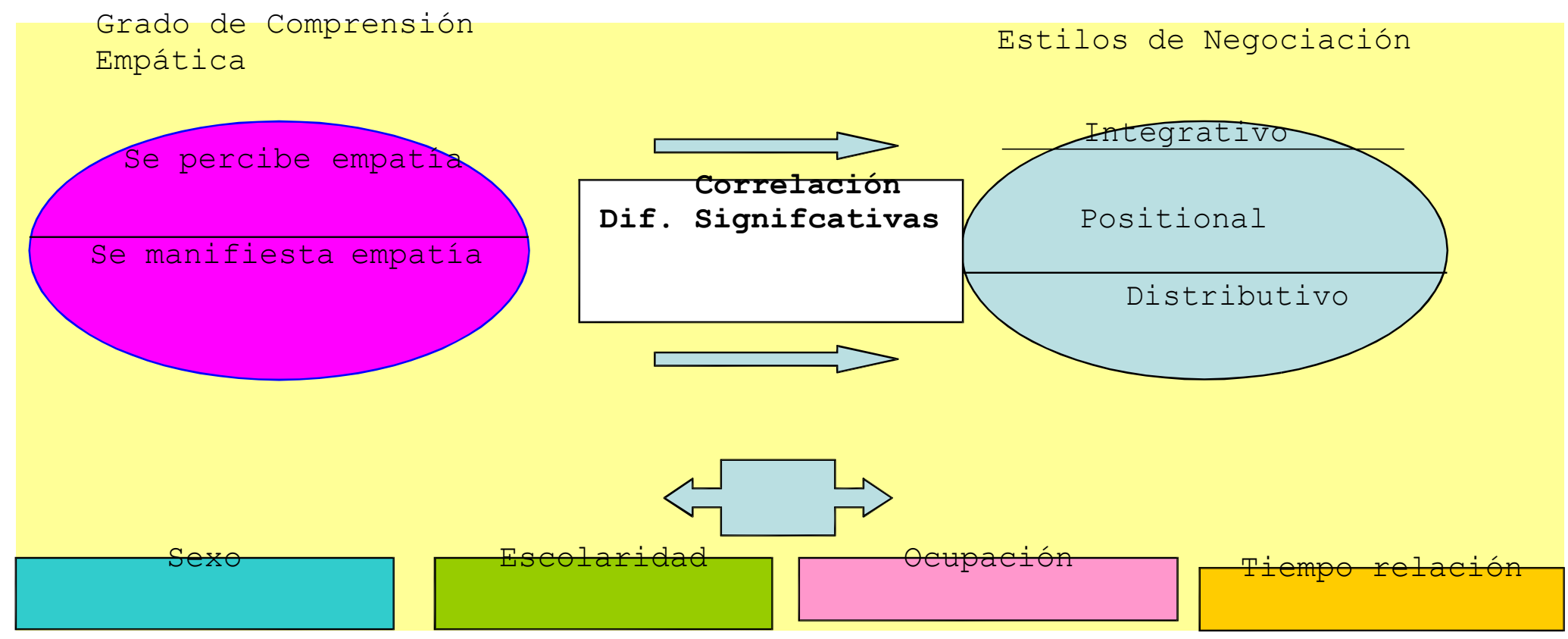


Tabla 2. Descripción por sexo.

\begin{tabular}{|c|c|c|c|}
\hline sexo & Media & Valor $\mathrm{t}$ & Simnificonoi \\
\hline $\begin{array}{ll}\text { Integradora } & \text { Hombre } \\
\text { Mujer } & \end{array}$ & $\begin{array}{l}4.1942 \\
3.9358\end{array}$ & $\begin{array}{l}1.250 \\
1.270\end{array}$ & $\begin{array}{l}.217 \\
.210\end{array}$ \\
\hline $\begin{array}{ll}\text { Posicional Hombre } & \text { Hujer }\end{array}$ & $\begin{array}{l}3.3152 \\
3.2407\end{array}$ & $\begin{array}{l}.465 \\
.465\end{array}$ & $\begin{array}{l}.644 \\
.644\end{array}$ \\
\hline $\begin{array}{ll}\text { Distributiva } & \text { Hombre } \\
\text { Mujer } & \end{array}$ & $\begin{array}{l}3.6522 \\
3.4630\end{array}$ & $\begin{array}{l}1.087 \\
1.090\end{array}$ & $\begin{array}{l}.282 \\
.281\end{array}$ \\
\hline $\begin{array}{l}\text { Me Empatiza Hombre } \\
\text { Mujer }\end{array}$ & $\begin{array}{l}2.9810 \\
3.2222\end{array}$ & $\begin{array}{l}-1.387 \\
-1.410\end{array}$ & $\begin{array}{l}.172 \\
.165\end{array}$ \\
\hline $\begin{array}{l}\text { Empatizo Hombre } \\
\text { Mujer }\end{array}$ & $\begin{array}{l}3.9701 \\
4.0347\end{array}$ & $\begin{array}{l}-.546 \\
-.535\end{array}$ & $\begin{array}{l}.587 \\
.595\end{array}$ \\
\hline
\end{tabular}


Tabla 3. Distribución por ocupación

\begin{tabular}{|l|c|c|c|c|}
\hline OCUPACION & $\begin{array}{l}\text { FEMENI } \\
\text { NO }\end{array}$ & MASCULINO & FRECUENCIA & PORCENTAJE \\
\hline Hogar & 4 & 0 & 4 & $8 \%$ \\
\hline Obrero & 1 & 2 & 3 & $6 \%$ \\
\hline Comerciante & 2 & 1 & 3 & $6 \%$ \\
\hline Profesionista & 20 & 20 & 40 & $80 \%$ \\
\hline Total & 27 & 23 & 50 & $100 \%$ \\
\hline
\end{tabular}

Figura 4. Análisis de Correlación

\section{Estilo Posicional Negociar}

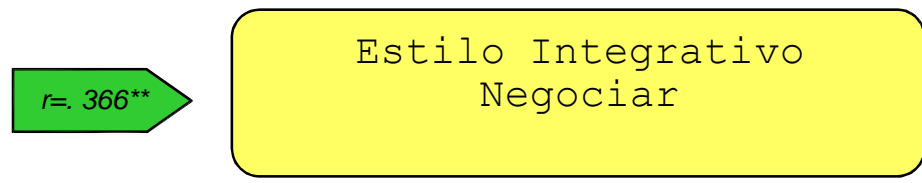

\section{(2) ( $)$}

Este texto está protegido por una licencia Creative Commons $\underline{4.0 .}$.

Usted es libre para Compartir — copiar y redistribuir el material en cualquier medio o formato- y Adaptar el documen- to remezclar, transformar y crear a partir del material - para cualquier propósito, incluso comercialmente, siempre que cumpla la condición de:

Atribución: Usted debe reconocer el crédito de una obra de manera adecuada, proporcionar un enlace a la licencia, e in- dicar si se han realizado cambios. Puede hacerlo en cualquier forma razonable, pero no de forma tal que sugiera que tie- ne el apoyo del licenciante o lo recibe por el uso que hace. 